\title{
Article
}

\author{
Doi 10.5943/sif/4/1/9
}

\section{Wild Mushrooms of Nagaland, India - An Important Bioresource}

\section{Ao $\mathbf{T}$ and Deb CR*}

Department of Botany, Nagaland University, Lumami 798 627, Nagaland, India

Ao T, Deb CR 2019 - Wild Mushrooms of Nagaland, India - An Important Bioresource. Studies in Fungi 4(1), 61-78, Doi 10.5943/sif/4/1/9

\begin{abstract}
The need for conservation of fungal diversity has garnered more interest during recent times. Nagaland is a hilly state of north-eastern region in India with varied altitude and agro-climatic conditions. The state is home to a large number of wild fungi including popular edible varieties and has the potential role to improve the rural economy. The present study, concerns with a total number of 141 mushroom species belonging to 80 genera under 44 families of which 52 species are identified to be edible, 10 species as poisonous and the remaining 79 mushrooms are found to be inedible. The rich mushroom wealth of the state is yet untapped so it is high time to study and explore this rich bioresource. This will create avenues for the local people to earn their livelihoods, and also create awareness for conservation and management of this bioresource. The database of wild mushrooms created in this study will help future researchers to make further in-depth study of the potential mushrooms available. Mushrooms are a boon to mankind and should be judiciously exploited for societal benefits across the globe.
\end{abstract}

Key words - Ascomycetes - basidiomycetes - biodiversity - edible - poisonous

\section{Introduction}

Conservation of macro-fungi has garnered additional interest during the last two decades (Chang \& Miles 2004). The existence of many fungal species is under threat due to climate change, urbanization, unsystematic exploration and collections, deforestation etc. Fungi are omnipresent, diverse, abundant and ecologically important. Recently, mushroom taxonomic and diversity studies have gained much importance because climate change and habitat destruction has affected the survivability of mushroom species in nature (Bhattacharjee et al. 2015). As estimated 70,000 species of fungi out of $\sim 1.5$ million fungi in existence are identified of which one third is said to be found in India (Wasser 2002, Manoharachary et al. 2005). Though the quantum of this important bioresource is very high, the diversity study is scanty.

The pre-historic collection of edible mushrooms for consumption started by trial and error and continued for a very long time (Atri et al. 1997). Since the early ages, mushrooms have been regarded around the globe as the most delectable and succulent of foods because of its unique taste and flavor (Chang \& Miles 1992). Therefore, mushrooms have been valued highly as a nutritious food by humans (Manzi et al. 1999). Besides, mushrooms are considered as ecological indicators and used in reforestation programs (Wongchalee \& Pukahute 2012, Andrew et al. 2013). Wild mushrooms are seasonal and one of the important non-wood forest products; provide seasonal food to rural people and also alternative source of income during the mushrooming season (Sysouphanthong et al. 2010). Though the demand for edible and medicinal mushrooms has 
increased over the years but research has not progressed accordingly. It is the need of the hour to explore and identify the wild mushroom resources as they are an integral partner in food security and checking environmental quality. The demand for mushrooms have increased due to awareness of the nutritional and therapeutic value of mushrooms and urges farmers to exploit different wild mushrooms. The nutritional value of wild edible mushrooms is considered higher then fish, meat and most vegetables including its medicinal benefits. Some of the most accepted edible mushrooms globally are Agaricus bisporus, Auricularia species, Pleurotus species, Lentinula edodes, Volvariella volvacea etc (Bonatti et al. 2004). Currently 270 mushroom species are known to have therapeutic properties and used as remedies for many diseases, as tonic and aphrodisiac (Wasser \& Weis 1999, Boa 2004, Tibuhwa 2013) but the therapeutic potential of mushrooms is yet fully untapped.

Nagaland, a small state of north-eastern region of India has rich mushroom biodiversity but still under studied. Wild edible mushrooms are highly favoured as a source of functional food in Nagaland which forms a vital part of the food culture of all the tribes in the state. The ethnic people collect the wild edible mushrooms species during the mushrooming season which starts from end of April to July for consumption as well as to sell them at the local markets. Since, the mushroom species are identified on the traditional knowledge, wrong identification of the species often leads to poisoning and death of the consuming populace. As mushrooms have become the most important horticultural crop in recent times, it is pertinent to know the mushroom wealth of the state for popularization and commercialization. Conservation of mushrooms can only begin with the knowledge about the food value and environmental challenges. Therefore, keeping in mind the importance of mushrooms, the objectives of the present work was to document the mushroom diversity of Nagaland and its scope.

\section{Materials \& Methods}

\section{Study Area}

Nagaland cover a total geographic area of $16,579 \mathrm{sq} \mathrm{km}$ and lies between $93^{\circ} 15^{\prime}$ to $95^{\circ} 15^{\prime} \mathrm{E}$ and $25^{\circ} 10^{\prime}$ to $27^{\circ} 4^{\prime} \mathrm{N}$. The calendar year can be divided into 4 seasons: winter (DecemberFebruary), pre-monsoon (March-April), monsoon (May-September) and retreating monsoon (October-November). The recorded average annual rainfall ranges between $2000-2500 \mathrm{~mm}$. The temperature during summer ranges from $16-34^{\circ} \mathrm{C}$ and drops as low as $4^{\circ} \mathrm{C}$ during winter. The state forest area is broadly classified into three forest groups based on climatic conditions and altitudes as Tropical, Sub-tropical and Temperate types which are further sub-divided into 9 major types:

1. Tropical forests - Tropical Wet Evergreen Forest, Tropical Semi- Evergreen Forest, Tropical Moist Evergreen Forest (up to1000m).

2. Sub-Tropical Forests - Sub-Tropical Evergreen Broadleaved Forests, Sub-Tropical Mixed Deciduous Broadleaved Forests, Sub- Tropical Alpine Forests (between 1000-1800m).

3. Temperate Forests - Montane Wet Temperate Forests, Temperate Rhododendron Forests, Sub- Alpine Forests (between 1800-3848m).

\section{Sample Collection}

Samples were collected from various parts of Nagaland throughout the year. The morphological characters like fruiting body color; size, shape and color of the stipe, pileus and lamellae position; presence of annulus; spore print, type of volva, its habitat, host and location of the mushrooms were recorded after collection and prior to preservation of the mushroom specimens in formaldehyde solution. Phenotypic identification of the collected mushroom samples was done based on morphological characters. Spores were collected through spore prints and observed under microscope to study the spore morphology. The specimens were preserved in $2 \%(\mathrm{v} / \mathrm{v})$ formaldehyde solution and maintained as herbarium for future references. All the voucher specimens were deposited in the herbarium of Department of Botany, Nagaland University, Lumami, India. For identification of mushrooms, standard manuals and keys were consulted 
(Crawshay 1930, Christensen 1943, Pegler 1983, Roy \& De 1996, Das \& Sharma 2005, Das 2009, Mortimer et al. 2014, Philips 2006). Mycokeys at www.mushroomexpert.com and www.mycokey.com were also consulted for identification. The local markets were surveyed during the season of availability to gather information on the wild mushroom varieties sold (Ao et al. 2016).

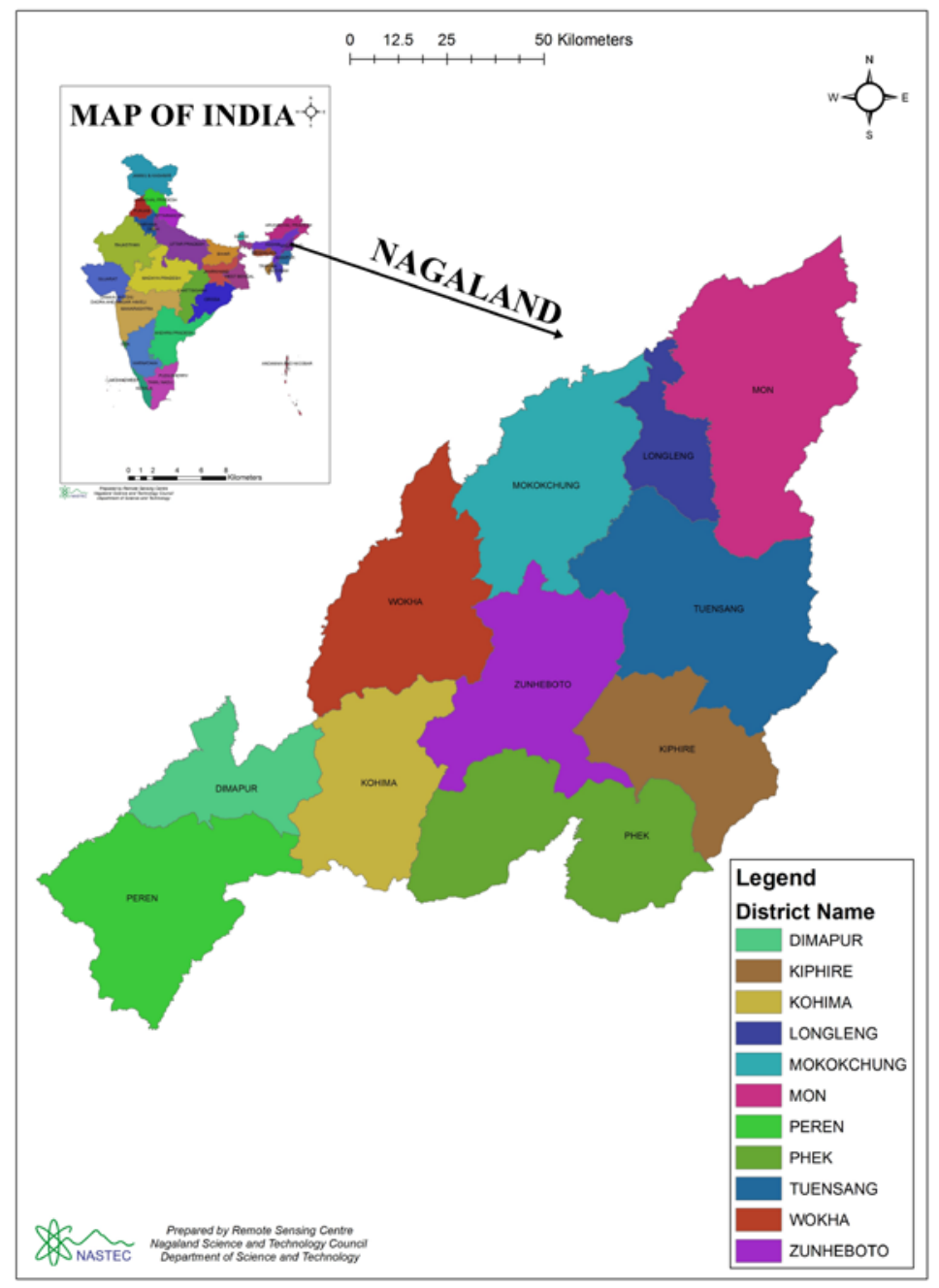

Fig. 1 - The map shows the geographical area of Nagaland.

\section{Results}

Nagaland has a forest cover of $75.33 \%$ of the state's geographical area according to India State of Forest Report, 2017. Due to various anthropogenic factors, forest cover is declining in the state which has exerted severe threat on the survivability of biodiversity. In the present study, wild mushrooms were collected from different areas of Nagaland covering altitudinal range of $150 \mathrm{~m}$ ASL to $3000 \mathrm{~m}$ ASL. It was observed that the mushroom populations decreased as the altitudinal range increased. A total number of 141 mushroom species belonging to 80 genera under 44 families - Russulaceae, Hygrophoraceae, Amanitaceae, Polyporaceae, Boletaceae, Agaricaceae, Lyophyllaceae, Auriculariaceae, Dacrymycetaceae, Inocybaceae, Suillaceae, Ganodermataceae, Tricholomataceae, Clavariaceae, Pleurotaceae, Tremellaceae, Omphalotaceae, Hericiaceae, Hymenochaetaceae, Psathyrellaceae, Cortinariaceae, Sclerodermataceae, Hydnangiaceae, Mycenaceae, Strophariaceae, Gomphaceae, Geastraceae, Phallaceae, Pyronemataceae, Leotiaceae, Xylariaceae, Clavicipitaceae, Schizophyllaceae, Nidulariaceae, Sparassidaceae, Physalacriaceae, Exidiaceae, Bulgariaceae, Entolomataceae, Hymenogastraceae, Clavulinaceae, Helvellaceae, Sarcoscyphaceae and Cantharellaceae have been identified correctly in the present study (Table 1, 
Figs 2-5). Out of 141 mushroom species, only 12 species belonged to Ascomycetes class and the remaining mushrooms belonged to Basidiomycetes class. Thus, the field surveys revealed that the mushroom species belonging to Basidiomycetes dominates over Ascomycetes. For identification, morphological observations were carried out as described above and spores were collected through spore prints and observed under microscope. A total of 52 mushroom species were identified as edible, 10 mushrooms were found to be poisonous and the remaining 79 mushrooms were found to be inedible. Schizophyllum commune, Auricularia auricula-judae, A. polytricha, Lactifluus piperatus, Lf. volemus, Sceleroderma citrinum, Termitomyces heimii, Tremella fuciformis, Tricholoma imbricatum, Hygrocybe conica and Agaricus moelleri was found to be the most abundant during the season of occurrence (Ao et al. 2016 \& present study). The collected species were predominantly found to be parasitic, saprophytic and ecto-mycorrhizal in habitat.

\section{Discussion}

Though mushrooms were found to grow round the year, but healthy growth was observed during May-October. Mushrooms require specific micro-climatic conditions for its growth and during the present investigation; it was observed that the presence and abundance of mushrooms has decreased over time due to anthropogenic factors and climate change. For the formation of mushroom fruit body, climate plays an important factor and change in the morphology, distribution and abundance of fungi is often linked to climatic changes (Diez et al. 2013). Thus, mushrooms can be regarded as indicators of ecosystem damage. Macro-fungi are mostly found in the wet season than the dry season. The dry season is mostly dominated by polypore and bracket fungi like Trametes gibbosa, T. versicolor, Pycnoporus cinnabarinus etc; this is due to decreased rainfall and increase in temperature where fleshy macro-fungi are not able to withstand these conditions (Andrew et al. 2013). Ao et al. (2016) reported the market status of few wild edible mushroom species in different markets of Nagaland. It was found that mushrooms are mostly sold in fresh as there is no proper post harvest processing and packaging facility available at present. Besides edible species, a good number of species are reported to be medicinally important e.g., Auricularia auricula-judae, A. polytricha, Lentinula edodes, Schizophyllum commune, Trametes gibbosa, $T$. versicolor, Pycnoporus cinnabarinus, Microporus xanthopus, Coprinus disseminatus, Daldinia concentrica, Ganoderma lucidum, Aleuria aurantia, Cantharellus cibarius, Hygrocybe conica, Lentinus squarrosulus, L. sajor-caju, Pleurotus pulmonarius, Lycoperdon perlatum, Flammulina velutipes, Cordyceps militaris, Tremella mesenterica, T. fuciformis (Chang \& Miles 2004, Wani et al. 2010, Ao et al. 2016). Ao \& Deb (2019) analyzed the nutritional and antioxidant potential of 10 wild edible mushrooms of Nagaland.

There has been significant progress in mushroom research because of nutritional and medicinal properties. The health benefits of consuming mushrooms and mushroom products have been reported by many workers around the world (Ferreira et al. 2007). Mushrooms are taken as functional food and as a source to develop drugs and nutraceuticals as they possess antioxidant, antitumor and antimicrobial properties (Lakhanpal \& Rana 2005). Besides pharmacological properties, mushrooms have become important in human diet due to rich nutritional value, related to high protein and low fat. These wild edible mushrooms are healthy food supplements required to maintain balanced diets as it contains macro as well as micro nutrients and functional minerals. Recently, mushrooms are advocated as a protein supplementary food and also as a low caloric diet food especially for cancer, diabetic and cardiac patients. The State of Food Security and Nutrition in the World (SOFI) has called upon all countries and stakeholders to act together to end hunger and prevent all forms of malnutrition by 2030 as the present scenario is that the number of undernourished people in the world is increasing alarmingly.

In addition to all this, gathering mushrooms from the wild or cultivating and selling gives employment opportunities to the rural people. The spent compost left after mushroom cultivation can also be used as manure. Moreover, thorough explorations of the wild mushrooms are required to study its medicinal properties. The present study revealed that Nagaland is home to a large number of wild mushrooms and proper measures need to be taken for sustainable utilization of this 
bioresource. To achieve the goal, there is the need to identify the rich mushroom biodiversity of the state which will help in creating strategies for management and conservation as many factors are affecting the survivability of mushrooms in nature. For the prosperity of society at large, wild edible mushrooms need to be domesticated for sustainable and commercial production.

Table 1 List of wild mushrooms collected and identified during the study

\begin{tabular}{|c|c|c|c|c|}
\hline Mushroom Species & Family & Habitat and Availability Status & Edibility & $\begin{array}{l}\text { Accession } \\
\text { No. } \\
\end{array}$ \\
\hline $\begin{array}{l}\text { Agaricus augustus } \\
\text { Fr. }\end{array}$ & Agaricaceae & $\begin{array}{l}\text { Found with deciduous or } \\
\text { coniferous trees, occasional. }\end{array}$ & Edible & $\begin{array}{l}\text { NUBOT-TA- } \\
\text { AA-95 }\end{array}$ \\
\hline $\begin{array}{l}\text { Agaricus moelleri } \\
\text { Wasser }\end{array}$ & Agaricaceae & $\begin{array}{l}\text { Under sub-tropical semi- } \\
\text { evergreen forests, occasional. }\end{array}$ & Poisonous & $\begin{array}{l}\text { NUBOT-TA- } \\
\text { AM-59 }\end{array}$ \\
\hline $\begin{array}{l}\text { Agaricus } \\
\text { xanthodermus } \\
\text { Genev. }\end{array}$ & Agaricaceae & $\begin{array}{l}\text { Found with deciduous or } \\
\text { coniferous trees, meadows or } \\
\text { gardens, occasional, vulnerable } \\
\text { on Red Data List. }\end{array}$ & Poisonous & $\begin{array}{l}\text { NUBOT-TA- } \\
\text { AX-140 }\end{array}$ \\
\hline $\begin{array}{l}\text { Coprinus comatus } \\
\text { (O.F. Müll.) Pers. }\end{array}$ & Agaricaceae & $\begin{array}{l}\text { Grows singly or in clusters or } \\
\text { lines on lawns, wood pieces or on } \\
\text { ground, common. }\end{array}$ & Edible & $\begin{array}{l}\text { NUBOT-TA- } \\
\text { CC-74 }\end{array}$ \\
\hline $\begin{array}{l}\text { Lepiota cristata } \\
\text { (Bolton) P. Kumm. }\end{array}$ & Agaricaceae & $\begin{array}{l}\text { Found in woods and leaf litter, } \\
\text { very common. }\end{array}$ & Inedible & $\begin{array}{l}\text { NUBOT-TA- } \\
\text { LC-57 }\end{array}$ \\
\hline $\begin{array}{l}\text { Lepiota felina } \\
\text { (Pers.) P. Karst. }\end{array}$ & Agaricaceae & Found with conifers, uncommon. & Poisonous & $\begin{array}{l}\text { NUBOT-TA- } \\
\text { LF-103 }\end{array}$ \\
\hline $\begin{array}{l}\text { Leucocoprinus } \\
\text { birnbaumii (Corda) } \\
\text { Singer }\end{array}$ & Agaricaceae & $\begin{array}{l}\text { Found in tropical areas, } \\
\text { uncommon. }\end{array}$ & Inedible & $\begin{array}{l}\text { NUBOT-TA- } \\
\text { LB-104 }\end{array}$ \\
\hline $\begin{array}{l}\text { Lepiota lilacea } \\
\text { Bres. }\end{array}$ & Agaricaceae & $\begin{array}{l}\text { Found with deciduous and } \\
\text { conifers, uncommon. }\end{array}$ & Poisonous & $\begin{array}{l}\text { NUBOT-TA- } \\
\text { LL-105 }\end{array}$ \\
\hline $\begin{array}{l}\text { Leucocoprinus } \\
\text { brebissonii (Godey) } \\
\text { Locq. }\end{array}$ & Agaricaceae & $\begin{array}{l}\text { Found in deciduous woods and } \\
\text { flowerpots, occasional. }\end{array}$ & Inedible & $\begin{array}{l}\text { NUBOT-TA- } \\
\text { LB-106 }\end{array}$ \\
\hline $\begin{array}{l}\text { Leucocoprinus } \\
\text { fragilissimus (Berk. } \\
\text { \& M. A. Curtis) } \\
\text { Pat. }\end{array}$ & Agaricaceae & $\begin{array}{l}\text { Found singly or scattered, in } \\
\text { humus or litter, in woods, } \\
\text { frequent. }\end{array}$ & Inedible & $\begin{array}{l}\text { NUBOT-TA- } \\
\text { LF-107 }\end{array}$ \\
\hline $\begin{array}{l}\text { Lycoperdon } \\
\text { perlatum Pers. }\end{array}$ & Agaricaceae & $\begin{array}{l}\text { Grows in fields, roadsides, in } \\
\text { woods and amongst fallen leaf } \\
\text { litter in mixed wood and conifers, } \\
\text { common. }\end{array}$ & $\begin{array}{l}\text { Edible } \\
\text { when } \\
\text { young and } \\
\text { white in } \\
\text { color }\end{array}$ & $\begin{array}{l}\text { NUBOT-TA- } \\
\text { LP-72 }\end{array}$ \\
\hline $\begin{array}{l}\text { Lycoperdon } \\
\text { pyriforme Schaeff. }\end{array}$ & Agaricaceae & $\begin{array}{l}\text { Grows on deadwood of } \\
\text { hardwoods or conifers, found } \\
\text { scattered or in dense clusters, } \\
\text { occasional. }\end{array}$ & Edible & $\begin{array}{l}\text { NUBOT-TA- } \\
\text { LP-89 }\end{array}$ \\
\hline $\begin{array}{l}\text { Macrolepiota } \\
\text { albuminosa (Berk.) } \\
\text { Pegler }\end{array}$ & Agaricaceae & $\begin{array}{l}\text { Grows on termite mounds in } \\
\text { grassy fields, common. }\end{array}$ & Edible & $\begin{array}{l}\text { NUBOT-TA- } \\
\text { MA-63 }\end{array}$ \\
\hline $\begin{array}{l}\text { Auricularia } \\
\text { auricula-judae } \\
\text { (Bull.) Quél. }\end{array}$ & Auriculariaceae & $\begin{array}{l}\text { On dead stumps and branches of } \\
\text { sub-tropical and temperate trees } \\
\text { especially Alnus. Grows on both } \\
\text { dead and living trees, very } \\
\text { common. }\end{array}$ & Edible & $\begin{array}{l}\text { NUBOT-TA- } \\
\text { AA-01 }\end{array}$ \\
\hline $\begin{array}{l}\text { Auricularia } \\
\text { mesenterica } \\
\text { (Dicks.) Pers. }\end{array}$ & Auriculariaceae & $\begin{array}{l}\text { On dead stumps and wood logs, } \\
\text { frequent. }\end{array}$ & Inedible & $\begin{array}{l}\text { NUBOT-TA- } \\
\text { AM-31 }\end{array}$ \\
\hline
\end{tabular}


Table 1 Continued.

\begin{tabular}{|c|c|c|c|c|}
\hline Mushroom Species & Family & Habitat and Availability Status & Edibility & $\begin{array}{l}\text { Accession } \\
\text { No. } \\
\end{array}$ \\
\hline $\begin{array}{l}\text { Auricularia } \\
\text { polytricha (Mont.) } \\
\text { Sacc. }\end{array}$ & Auriculariaceae & $\begin{array}{l}\text { In clusters on rotten or dead and } \\
\text { decaying stumps and twigs, very } \\
\text { common. }\end{array}$ & Edible & $\begin{array}{l}\text { NUBOT-TA- } \\
\text { AP-02 }\end{array}$ \\
\hline $\begin{array}{l}\text { Lactarius deliciosus } \\
\text { (L.) Gray }\end{array}$ & Russulaceae & $\begin{array}{l}\text { Found under sub-tropical semi- } \\
\text { evergreen forests including pine, } \\
\text { frequent. }\end{array}$ & Edible & $\begin{array}{l}\text { NUBOT-TA- } \\
\text { LD-112 }\end{array}$ \\
\hline $\begin{array}{l}\text { Lactarius } \\
\text { deterrimus Gröger }\end{array}$ & Russulaceae & $\begin{array}{l}\text { Found under sub-tropical semi- } \\
\text { evergreen forests, frequent. }\end{array}$ & Edible & $\begin{array}{l}\text { NUBOT-TA- } \\
\text { LD-114 }\end{array}$ \\
\hline $\begin{array}{l}\text { Lactifluus } \\
\text { glaucescens } \\
\text { (Crossl.) Verbeken }\end{array}$ & Russulaceae & $\begin{array}{l}\text { Under Quercus and Lithocarpus } \\
\text { in sub-tropical forests, frequent. }\end{array}$ & Inedible & $\begin{array}{l}\text { NUBOT-TA- } \\
\text { LG-83 }\end{array}$ \\
\hline $\begin{array}{l}\text { Lactifluus piperatus } \\
\text { (L.) Pers. }\end{array}$ & Russulaceae & $\begin{array}{l}\text { Under sub-tropical semi- } \\
\text { evergreen forests, common. }\end{array}$ & Edible & $\begin{array}{l}\text { NUBOT-TA- } \\
\text { LP-04 }\end{array}$ \\
\hline $\begin{array}{l}\text { Lactarius subdulcis } \\
\text { (Pers.) Gray }\end{array}$ & Russulaceae & $\begin{array}{l}\text { Found in woods mostly near } \\
\text { broad leaved trees, common }\end{array}$ & Edible & $\begin{array}{l}\text { NUBOT-TA- } \\
\text { LS-136 }\end{array}$ \\
\hline $\begin{array}{l}\text { Lactifluus volemus } \\
\text { (Fr.) Kuntze }\end{array}$ & Russulaceae & $\begin{array}{l}\text { Under sub-tropical semi- } \\
\text { evergreen forests including pine, } \\
\text { common. }\end{array}$ & Edible & $\begin{array}{l}\text { NUBOT-TA- } \\
\text { LV-05 }\end{array}$ \\
\hline $\begin{array}{l}\text { Russula } \\
\text { cyanoxantha } \\
\text { (Schaeff.) Fr. }\end{array}$ & Russulaceae & $\begin{array}{l}\text { Found under Lithocarpus and } \\
\text { Castanopsis in sub-tropical } \\
\text { forests, frequent. }\end{array}$ & Inedible & $\begin{array}{l}\text { NUBOT-TA- } \\
\text { RC-36 }\end{array}$ \\
\hline Russula fragilis Fr. & Russulaceae & $\begin{array}{l}\text { Found under Lithocarpus and } \\
\text { Castanopsis in sub-tropical } \\
\text { forests and conifers, common. }\end{array}$ & Inedible & $\begin{array}{l}\text { NUBOT-TA- } \\
\text { RF-42 }\end{array}$ \\
\hline $\begin{array}{l}\text { Russula } \\
\text { heterophylla (Fr.) } \\
\text { Fr. }\end{array}$ & Russulaceae & $\begin{array}{l}\text { Found under Lithocarpus and } \\
\text { Castanopsis in sub-tropical } \\
\text { forests, occasional. }\end{array}$ & Edible & $\begin{array}{l}\text { NUBOT-TA- } \\
\text { RH-44 }\end{array}$ \\
\hline $\begin{array}{l}\text { Russula nobilis } \\
\text { Velen }\end{array}$ & Russulaceae & $\begin{array}{l}\text { Found under Lithocarpus and } \\
\text { Castanopsis in sub-tropical } \\
\text { forests, common. }\end{array}$ & Poisonous & $\begin{array}{l}\text { NUBOT-TA- } \\
\text { RN-43 }\end{array}$ \\
\hline $\begin{array}{l}\text { Russsula } \\
\text { natarajanii K. Das, } \\
\text { J.R. Sharma \& Atri }\end{array}$ & Russulaceae & $\begin{array}{l}\text { Found under Lithocarpus and } \\
\text { Castanopsis in sub-tropical } \\
\text { forests, occasional. }\end{array}$ & Inedible & $\begin{array}{l}\text { NUBOT-TA- } \\
\text { RN-11 }\end{array}$ \\
\hline $\begin{array}{l}\text { Russula ochroleuca } \\
\text { Fr. }\end{array}$ & Russulaceae & $\begin{array}{l}\text { Found with conifers, very } \\
\text { common }\end{array}$ & Edible & $\begin{array}{l}\text { NUBOT-TA- } \\
\text { RO-101 }\end{array}$ \\
\hline $\begin{array}{l}\text { Russula puellaris } \\
\text { Fr. }\end{array}$ & Russulaceae & Found with conifers, frequent. & Edible & $\begin{array}{l}\text { NUBOT-TA- } \\
\text { RP-102 }\end{array}$ \\
\hline Russula rosea Pers. & Russulaceae & $\begin{array}{l}\text { Found near deciduous trees like } \\
\text { beech, frequent. }\end{array}$ & Inedible & $\begin{array}{l}\text { NUBOT-TA- } \\
\text { RR-100 }\end{array}$ \\
\hline $\begin{array}{l}\text { Rusulla senecis } \mathrm{S} . \\
\text { Imai }\end{array}$ & Russulaceae & $\begin{array}{l}\text { Found under Lithocarpus and } \\
\text { Castanopsis in sub-tropical } \\
\text { forests, frequent. }\end{array}$ & $\begin{array}{l}\text { Edible but } \\
\text { not } \\
\text { consumed } \\
\text { by the } \\
\text { locals }\end{array}$ & $\begin{array}{l}\text { NUBOT-TA- } \\
\text { RS-10 }\end{array}$ \\
\hline Russula sp. & Russulaceae & $\begin{array}{l}\text { Found with deciduous and broad- } \\
\text { leaved trees, frequent. }\end{array}$ & Inedible & $\begin{array}{l}\text { NUBOT-TA- } \\
\text { R-68 }\end{array}$ \\
\hline
\end{tabular}


Table 1 Continued.

\begin{tabular}{|c|c|c|c|c|}
\hline Mushroom Species & Family & Habitat and Availability Status & Edibility & $\begin{array}{l}\text { Accession } \\
\text { No. }\end{array}$ \\
\hline $\begin{array}{l}\text { Ganoderma } \\
\text { applanatum (Pers.) } \\
\text { Pat. }\end{array}$ & $\begin{array}{l}\text { Ganodermatace } \\
\text { ae }\end{array}$ & $\begin{array}{l}\text { On trunks and roots of trees, } \\
\text { occasional. }\end{array}$ & Inedible & $\begin{array}{l}\text { NUBOT-TA- } \\
\text { GA-80 }\end{array}$ \\
\hline $\begin{array}{l}\text { Ganoderma } \\
\text { lucidum (Curtis) P. } \\
\text { Karst. }\end{array}$ & $\begin{array}{l}\text { Ganodermatace } \\
\text { ae }\end{array}$ & $\begin{array}{l}\text { On trunks and roots of Quercus } \\
\text { species, uncommon. }\end{array}$ & Inedible & $\begin{array}{l}\text { NUBOT-TA- } \\
\text { GL-20 }\end{array}$ \\
\hline $\begin{array}{l}\text { Ramaria stricta } \\
\text { (Pers.) Quél }\end{array}$ & Gomphaceae & $\begin{array}{l}\text { Grows on ground in association } \\
\text { with dead tree stumps, frequent, } \\
\text { Vulnerable on Red Data List. }\end{array}$ & Inedible & $\begin{array}{l}\text { NUBOT-TA- } \\
\text { RS-67 }\end{array}$ \\
\hline $\begin{array}{l}\text { Cantharellus } \\
\text { cibarius (Fr.) }\end{array}$ & $\begin{array}{l}\text { Cantharellacea } \\
\text { e }\end{array}$ & $\begin{array}{l}\text { Found under Lithocarpus in sub- } \\
\text { tropical forests, frequent. }\end{array}$ & Edible & $\begin{array}{l}\text { NUBOT-TA- } \\
\text { CC-03 }\end{array}$ \\
\hline $\begin{array}{l}\text { Lentinula edodes } \\
\text { (Berk.) Pegler }\end{array}$ & Omphalotaceae & On trunks of Oak trees, common. & Edible & $\begin{array}{l}\text { NUBOT-TA- } \\
\text { LE-06 }\end{array}$ \\
\hline $\begin{array}{l}\text { Marasmiellus } \\
\text { candidus (Fr.) } \\
\text { Singer }\end{array}$ & Omphalotaceae & $\begin{array}{l}\text { Found on fallen branches, } \\
\text { amongst leaf litter, uncommon }\end{array}$ & Inedible & $\begin{array}{l}\text { NUBOT-TA- } \\
\text { MC-138 }\end{array}$ \\
\hline $\begin{array}{l}\text { Marasmiellus } \\
\text { ramealis (Bull.) } \\
\text { Singer }\end{array}$ & Omphalotaceae & $\begin{array}{l}\text { Found on old stems, broken } \\
\text { twigs, common. }\end{array}$ & Inedible & $\begin{array}{l}\text { NUBOT-TA- } \\
\text { MR-137 }\end{array}$ \\
\hline $\begin{array}{l}\text { Calocera viscosa } \\
\text { (Pers.) Fr. }\end{array}$ & $\begin{array}{l}\text { Dacrymycetace } \\
\text { ae }\end{array}$ & $\begin{array}{l}\text { Grows on dead stumps in } \\
\text { temperate evergreen forests, } \\
\text { uncommon. }\end{array}$ & Inedible & $\begin{array}{l}\text { NUBOT-TA- } \\
\text { CV-25 }\end{array}$ \\
\hline $\begin{array}{l}\text { Dacrymyces } \\
\text { chrysospermus } \\
\text { Berk. \& M.A. } \\
\text { Curtis }\end{array}$ & $\begin{array}{l}\text { Dacrymycetace } \\
\text { ae }\end{array}$ & $\begin{array}{l}\text { Found under coniferous woods, } \\
\text { uncommon. }\end{array}$ & Inedible & $\begin{array}{l}\text { NUBOT-TA- } \\
\text { DC-129 }\end{array}$ \\
\hline $\begin{array}{l}\text { Dacrymyces } \\
\text { stillatus Nees }\end{array}$ & $\begin{array}{l}\text { Dacrymycetace } \\
\text { ae }\end{array}$ & $\begin{array}{l}\text { On damp decaying wood, } \\
\text { frequent. }\end{array}$ & Inedible & $\begin{array}{l}\text { NUBOT-TA- } \\
\text { DS-52 }\end{array}$ \\
\hline $\begin{array}{l}\text { Hericium cirrhatum } \\
\text { (Pers.) Nikol. }\end{array}$ & Hericiaceae & $\begin{array}{l}\text { On trunks of semi-evergreen and } \\
\text { temperate trees, uncommon, } \\
\text { vulnerable on Red Data List. }\end{array}$ & Edible & $\begin{array}{l}\text { NUBOT-TA- } \\
\text { HC-07 }\end{array}$ \\
\hline $\begin{array}{l}\text { Hericium erinaceus } \\
\text { (Bull.) Pers. }\end{array}$ & Hericiaceae & $\begin{array}{l}\text { Found on living deciduous trees, } \\
\text { occasional, vulnerable on Red } \\
\text { Data List. }\end{array}$ & Edible & $\begin{array}{l}\text { NUBOT-TA- } \\
\text { HE-132 }\end{array}$ \\
\hline $\begin{array}{l}\text { Schizophyllum } \\
\text { commune Fr. }\end{array}$ & $\begin{array}{l}\text { Schizophyllace } \\
\text { ae }\end{array}$ & $\begin{array}{l}\text { On branches of dead wood and } \\
\text { cut timber, very common. }\end{array}$ & Edible & $\begin{array}{l}\text { NUBOT-TA- } \\
\text { SC-09 }\end{array}$ \\
\hline Geastrum sp. & Geastraceae & In coniferous forests, occasional. & Inedible & $\begin{array}{l}\text { NUBOT-TA- } \\
\text { G-82 }\end{array}$ \\
\hline $\begin{array}{l}\text { Hymenochaete } \\
\text { rubiginosa (Dicks.) } \\
\text { Lev. }\end{array}$ & $\begin{array}{l}\text { Hymenochaeta } \\
\text { ceae }\end{array}$ & $\begin{array}{l}\text { On old rotting tree stumps, } \\
\text { uncommon. }\end{array}$ & Inedible & $\begin{array}{l}\text { NUBOT-TA- } \\
\text { HR-15 }\end{array}$ \\
\hline $\begin{array}{l}\text { Aureoboletus } \\
\text { auriporus (Peck) } \\
\text { Pouzar }\end{array}$ & Boletaceae & $\begin{array}{l}\text { Under sub-tropical semi- } \\
\text { evergreen forests, frequent. }\end{array}$ & Inedible & $\begin{array}{l}\text { NUBOT-TA- } \\
\text { AA-53 }\end{array}$ \\
\hline Boletus sp. & Boletaceae & $\begin{array}{l}\text { Under Castanopsis and } \\
\text { Lithocarpus in sub-tropical } \\
\text { forests }\end{array}$ & Inedible & $\begin{array}{l}\text { NUBOT-TA- } \\
\text { B-70 }\end{array}$ \\
\hline Boletus edulis Bull. & Boletaceae & $\begin{array}{l}\text { Under coniferous and semi- } \\
\text { evergreen forest types, } \\
\text { occasional. }\end{array}$ & Edible & $\begin{array}{l}\text { NUBOT-TA- } \\
\text { BE-22 }\end{array}$ \\
\hline
\end{tabular}


Table 1 Continued.

\begin{tabular}{|c|c|c|c|c|}
\hline Mushroom Species & Family & Habitat and Availability Status & Edibility & $\begin{array}{l}\text { Accession } \\
\text { No. } \\
\end{array}$ \\
\hline $\begin{array}{l}\text { Strobilomyces } \\
\text { strobilaceus. } \\
\text { (Scop.) Berk }\end{array}$ & Boletaceae & $\begin{array}{l}\text { Grows in association with semi- } \\
\text { evergreen and coniferous trees, } \\
\text { uncommon. }\end{array}$ & Edible & $\begin{array}{l}\text { NUBOT-TA- } \\
\text { SS-12 }\end{array}$ \\
\hline $\begin{array}{l}\text { Xerocomellus } \\
\text { chrysenteron } \\
\text { (Bull.) Šutara }\end{array}$ & Boletaceae & $\begin{array}{l}\text { Under sub-tropical semi- } \\
\text { evergreen forests including pine, } \\
\text { frequent. }\end{array}$ & Edible & $\begin{array}{l}\text { NUBOT-TA- } \\
\text { XC-48 }\end{array}$ \\
\hline $\begin{array}{l}\text { Cerioporus } \\
\text { leptocephalus } \\
\text { (Jacq.) Zmitr. }\end{array}$ & Polyporaceae & $\begin{array}{l}\text { Found under dead and decaying } \\
\text { tree stumps, common. }\end{array}$ & Inedible & $\begin{array}{l}\text { NUBOT-TA- } \\
\text { CL-131 }\end{array}$ \\
\hline $\begin{array}{l}\text { Laetiporus } \\
\text { sulphureus (Bull.) } \\
\text { Murr. }\end{array}$ & Polyporaceae & $\begin{array}{l}\text { Grows on dead stumps as well as } \\
\text { living tree trunk of hardwoods } \\
\text { and oaks, uncommon. }\end{array}$ & Edible & $\begin{array}{l}\text { NUBOT-TA- } \\
\text { LS-73 }\end{array}$ \\
\hline $\begin{array}{l}\text { Lentinus sajor-caju } \\
\text { (Fr.) Fr. }\end{array}$ & Polyporaceae & $\begin{array}{l}\text { Grows on dead stumps of trees } \\
\text { like Oak, common. }\end{array}$ & Edible & $\begin{array}{l}\text { NUBOT-TA- } \\
\text { LS-87 }\end{array}$ \\
\hline $\begin{array}{l}\text { Lentinus } \\
\text { squarrosulus Mont. } \\
\text { Singer }\end{array}$ & Polyporaceae & $\begin{array}{l}\text { On dead stumps of trees like Oak, } \\
\text { frequent. }\end{array}$ & Edible & $\begin{array}{l}\text { NUBOT-TA- } \\
\text { LS-40 }\end{array}$ \\
\hline $\begin{array}{l}\text { Lentinus tigrinus } \\
\text { (Bull.) Fr. }\end{array}$ & Polyporaceae & $\begin{array}{l}\text { Grows on dead stumps of trees } \\
\text { like Oak, frequent. }\end{array}$ & Edible & $\begin{array}{l}\text { NUBOT-TA- } \\
\text { LT-69 }\end{array}$ \\
\hline $\begin{array}{l}\text { Microporus } \\
\text { xanthopus (Fr.) } \\
\text { Kuntze }\end{array}$ & Polyporaceae & $\begin{array}{l}\text { Grows on fallen branches and } \\
\text { twigs, very common. }\end{array}$ & Inedible & $\begin{array}{l}\text { NUBOT-TA- } \\
\text { MX-14 }\end{array}$ \\
\hline $\begin{array}{l}\text { Pcynoporus } \\
\text { cinnabarinus } \\
\text { (Jacq.) P. Karst. }\end{array}$ & Polyporaceae & $\begin{array}{l}\text { Grows on fallen, dead and } \\
\text { decaying stumps of trees like } \\
\text { Alnus, common. }\end{array}$ & Inedible & $\begin{array}{l}\text { NUBOT-TA- } \\
\text { PC-13 }\end{array}$ \\
\hline $\begin{array}{l}\text { Trametes gibbosa } \\
\text { (Pers.) Fr. }\end{array}$ & Polyporaceae & On dead tree stumps, common. & Inedible & $\begin{array}{l}\text { NUBOT-TA- } \\
\text { TG-16 }\end{array}$ \\
\hline $\begin{array}{l}\text { Trametes hirsuta } \\
\text { (Wilfen) Pilat }\end{array}$ & Polyporaceae & On dead tree stumps, common. & Inedible & $\begin{array}{l}\text { NUBOT-TA- } \\
\text { TH-17 }\end{array}$ \\
\hline $\begin{array}{l}\text { Trametes versicolor } \\
\text { (L.) Lloyd }\end{array}$ & Polyporaceae & $\begin{array}{l}\text { On dead and decaying tree } \\
\text { stumps, frequent. }\end{array}$ & Inedible & $\begin{array}{l}\text { NUBOT-TA- } \\
\text { TV-38 }\end{array}$ \\
\hline $\begin{array}{l}\text { Coprinus } \\
\text { disseminatus (Pers.) } \\
\text { J.E.Lange }\end{array}$ & Psathyrellaceae & $\begin{array}{l}\text { In troops around the stumps of } \\
\text { dead, decaying wood, frequent. }\end{array}$ & Inedible & $\begin{array}{l}\text { NUBOT-TA- } \\
\text { CD-18 }\end{array}$ \\
\hline $\begin{array}{l}\text { Coprinellus } \\
\text { micaceus (Bull.) } \\
\text { Vilgalys, Hopple \& } \\
\text { Jacq. Johnson }\end{array}$ & Psathyrellaceae & $\begin{array}{l}\text { Found in clusters in decaying } \\
\text { woods, common. }\end{array}$ & Edible & $\begin{array}{l}\text { NUBOT-TA- } \\
\text { CM-118 }\end{array}$ \\
\hline $\begin{array}{l}\text { Psathyrella } \\
\text { piluliformis (Bull.) } \\
\text { P.D. Orton }\end{array}$ & Psathyrellaceae & $\begin{array}{l}\text { Found in groups under dead or } \\
\text { decaying stumps in sub-tropical } \\
\text { forests, common. }\end{array}$ & Inedible & $\begin{array}{l}\text { NUBOT-TA- } \\
\text { PP-134 }\end{array}$ \\
\hline $\begin{array}{l}\text { Daldinia } \\
\text { concentrica } \\
\text { (Bolton) Cesati \& } \\
\text { de Notaris }\end{array}$ & Xylariaceae & On dead wood logs, frequent. & Inedible & $\begin{array}{l}\text { NUBOT-TA- } \\
\text { DC-21 }\end{array}$ \\
\hline $\begin{array}{l}\text { Xylaria hypoxylon } \\
\text { (L.) Grev. }\end{array}$ & Xylariaceae & $\begin{array}{l}\text { Found in groups under dead } \\
\text { woods, frequent. }\end{array}$ & Inedible & $\begin{array}{l}\text { NUBOT-TA- } \\
\text { XH-122 }\end{array}$ \\
\hline $\begin{array}{l}\text { Xylaria polymorpha } \\
\text { (Pers.) Grev. }\end{array}$ & Xylariaceae & $\begin{array}{l}\text { Found in groups under dead or } \\
\text { decaying woods, frequent. }\end{array}$ & Inedible & $\begin{array}{l}\text { NUBOT-TA- } \\
\text { XP-123 }\end{array}$ \\
\hline $\begin{array}{l}\text { Cortinarius } \\
\text { purpurascens Fr. }\end{array}$ & Cortinariaceae & $\begin{array}{l}\text { Grows in association with semi- } \\
\text { evergreen and coniferous woods, } \\
\text { occasional. }\end{array}$ & Inedible & $\begin{array}{l}\text { NUBOT-TA- } \\
\text { CP-23 }\end{array}$ \\
\hline
\end{tabular}


Table 1 Continued.

\begin{tabular}{|c|c|c|c|c|}
\hline Mushroom Species & Family & Habitat and Availability Status & Edibility & $\begin{array}{l}\text { Accession } \\
\text { No. }\end{array}$ \\
\hline $\begin{array}{l}\text { Scleroderma } \\
\text { areolatum Ehrenb. }\end{array}$ & $\begin{array}{l}\text { Sclerodermatac } \\
\text { eae }\end{array}$ & $\begin{array}{l}\text { Found in moist places on ground } \\
\text { under sub-tropical areas, } \\
\text { uncommon. }\end{array}$ & Inedible & $\begin{array}{l}\text { NUBOT-TA- } \\
\text { SA-108 }\end{array}$ \\
\hline $\begin{array}{l}\text { Scleroderma } \\
\text { citrinum Pers. }\end{array}$ & $\begin{array}{l}\text { Sclerodermatac } \\
\text { eae }\end{array}$ & $\begin{array}{l}\text { On mossy or peaty ground in any } \\
\text { forest type, common. }\end{array}$ & Inedible & $\begin{array}{l}\text { NUBOT-TA- } \\
\text { SC-24 }\end{array}$ \\
\hline $\begin{array}{l}\text { Arrhenia onisca } \\
\text { (Fr.) Redhead, } \\
\text { Lutzoni, Moncalvo } \\
\text { \& Vilgalys }\end{array}$ & $\begin{array}{l}\text { Tricholomatace } \\
\text { ae }\end{array}$ & $\begin{array}{l}\text { Found scattered in small groups } \\
\text { on ground in litter or moss, } \\
\text { occasional. }\end{array}$ & Inedible & $\begin{array}{l}\text { NUBOT-TA- } \\
\text { AO-115 }\end{array}$ \\
\hline $\begin{array}{l}\text { Lepista nuda (Bull.) } \\
\text { Cooke }\end{array}$ & $\begin{array}{l}\text { Tricholomatace } \\
\text { ae }\end{array}$ & $\begin{array}{l}\text { Found to grow under hardwoods } \\
\text { even in gardens, common. }\end{array}$ & Edible & $\begin{array}{l}\text { NUBOT-TA- } \\
\text { LN-130 }\end{array}$ \\
\hline $\begin{array}{l}\text { Melanoleuca } \\
\text { grammopodia } \\
\text { (Bull.) Murrill }\end{array}$ & $\begin{array}{l}\text { Tricholomatace } \\
\text { ae }\end{array}$ & $\begin{array}{l}\text { Grows on leaf mulch or } \\
\text { composted soil in fields, } \\
\text { common. }\end{array}$ & Edible & $\begin{array}{l}\text { NUBOT-TA- } \\
\text { MG-61 }\end{array}$ \\
\hline $\begin{array}{l}\text { Tricholoma } \\
\text { imbricatum (Fr.) P. } \\
\text { Kumm. }\end{array}$ & $\begin{array}{l}\text { Tricholomatace } \\
\text { ae }\end{array}$ & $\begin{array}{l}\text { In coniferous woods, especially } \\
\text { with pine, frequent. }\end{array}$ & Edible & $\begin{array}{l}\text { NUBOT-TA- } \\
\text { TI-27 }\end{array}$ \\
\hline $\begin{array}{l}\text { Clavaria fragilis } \\
\text { Holmsk. }\end{array}$ & Clavariaceae & $\begin{array}{l}\text { Grows in clusters on ground } \\
\text { amongst leaf litters and in fields, } \\
\text { common. }\end{array}$ & Edible & $\begin{array}{l}\text { NUBOT-TA- } \\
\text { CF-35 }\end{array}$ \\
\hline $\begin{array}{l}\text { Clavulinopsis } \\
\text { fusiformis } \\
\text { (Sowerby) Corner. }\end{array}$ & Clavariaceae & $\begin{array}{l}\text { Grows in tufts on ground amongst } \\
\text { grasses and leaf litters, frequent. }\end{array}$ & Inedible & $\begin{array}{l}\text { NUBOT-TA- } \\
\text { CF-34 }\end{array}$ \\
\hline $\begin{array}{l}\text { Cordyceps militaris } \\
\text { (L.) Fr. }\end{array}$ & Clavicipitaceae & $\begin{array}{l}\text { Grows singly on ground after } \\
\text { parasitizing on the larvae or } \\
\text { pupae of butterflies and moths, } \\
\text { frequent. }\end{array}$ & Inedible & $\begin{array}{l}\text { NUBOT-TA- } \\
\text { CM-26 }\end{array}$ \\
\hline $\begin{array}{l}\text { Crucibulum laeve } \\
\text { (Huds.) Kambly }\end{array}$ & Nidulariaceae & $\begin{array}{l}\text { On twigs, fallen branches and } \\
\text { other vegetable remains, frequent. }\end{array}$ & Inedible & $\begin{array}{l}\text { NUBOT-TA- } \\
\text { CL-30 }\end{array}$ \\
\hline $\begin{array}{l}\text { Leotia lubrica } \\
\text { (Scop.) Pers. }\end{array}$ & Leotiaceae & $\begin{array}{l}\text { In damp areas in almost all forest } \\
\text { types, occasional. }\end{array}$ & Inedible & $\begin{array}{l}\text { NUBOT-TA- } \\
\text { LL-32 }\end{array}$ \\
\hline $\begin{array}{l}\text { Tremella fuciformis } \\
\text { Berk. }\end{array}$ & Tremellaceae & $\begin{array}{l}\text { On dead or fallen branches of } \\
\text { broadleaved trees, common. }\end{array}$ & Edible & $\begin{array}{l}\text { NUBOT-TA- } \\
\text { TF-37 }\end{array}$ \\
\hline $\begin{array}{l}\text { Tremella foliacea } \\
\text { Pers. }\end{array}$ & Tremellaceae & $\begin{array}{l}\text { Found on dead and decaying logs } \\
\text { like Lithocarpus, uncommon. }\end{array}$ & Inedible & $\begin{array}{l}\text { NUBOT-TA- } \\
\text { TF-116 }\end{array}$ \\
\hline $\begin{array}{l}\text { Tremella } \\
\text { mesenterica Retz. }\end{array}$ & Tremellaceae & Grows on dead woods, common. & Inedible & $\begin{array}{l}\text { NUBOT-TA- } \\
\text { TM-86 }\end{array}$ \\
\hline $\begin{array}{l}\text { Gloioxanthomyces } \\
\text { nitidus (Berk. \& } \\
\text { M.A. Curtis) } \\
\text { Lodge, Vizzini, } \\
\text { Ercole \& Boertm. }\end{array}$ & $\begin{array}{l}\text { Hygrophoracea } \\
\text { e }\end{array}$ & $\begin{array}{l}\text { Grows in clusters in woods and } \\
\text { damp soils, common. }\end{array}$ & Inedible & $\begin{array}{l}\text { NUBOT-TA- } \\
\text { GN-79 }\end{array}$ \\
\hline $\begin{array}{l}\text { Hygrocybe } \\
\text { cantharellus } \\
\text { (Schwein.) Murrill }\end{array}$ & $\begin{array}{l}\text { Hygrophoracea } \\
\text { e }\end{array}$ & $\begin{array}{l}\text { Grows in damp soils and moss, } \\
\text { frequent. }\end{array}$ & Inedible & $\begin{array}{l}\text { NUBOT-TA- } \\
\text { HC-85 }\end{array}$ \\
\hline $\begin{array}{l}\text { Hygrocybe conica } \\
\text { (Schaeff.) P. } \\
\text { Kumm. }\end{array}$ & $\begin{array}{l}\text { Hygrophoracea } \\
\text { e }\end{array}$ & $\begin{array}{l}\text { In grass fields after fresh burning } \\
\text { of forest, frequent. }\end{array}$ & Edible & $\begin{array}{l}\text { NUBOT-TA- } \\
\text { HC-41 }\end{array}$ \\
\hline $\begin{array}{l}\text { Hygrocybe miniata } \\
\text { (Fr.) P. Kumm. }\end{array}$ & $\begin{array}{l}\text { Hygrophoracea } \\
\text { e }\end{array}$ & $\begin{array}{l}\text { Grows in fields, woods or grassy } \\
\text { fields, common. }\end{array}$ & Ine & $\begin{array}{l}\text { NUBOT-TA- } \\
\text { HM-78 }\end{array}$ \\
\hline $\begin{array}{l}\text { Hygrocybe vitellina } \\
\text { (Fr.) P. Karst. }\end{array}$ & $\begin{array}{l}\text { Hygrophoracea } \\
\text { e }\end{array}$ & Amongst damp moss, frequent. & Inedible & $\begin{array}{l}\text { NUBOT-TA- } \\
\text { HV- } 47\end{array}$ \\
\hline
\end{tabular}


Table 1 Continued.

\begin{tabular}{|c|c|c|c|c|}
\hline Mushroom Species & Family & Habitat and Availability Status & Edibility & $\begin{array}{l}\text { Accession } \\
\text { No. } \\
\end{array}$ \\
\hline $\begin{array}{l}\text { Lichenomphalia } \\
\text { umbellifera (L.) } \\
\text { Redhead, Lutzoni, } \\
\text { Moncalvo \& } \\
\text { Vilgalys }\end{array}$ & $\begin{array}{l}\text { Hygrophoracea } \\
\text { e }\end{array}$ & $\begin{array}{l}\text { On fallen twigs in moist woods, } \\
\text { common. }\end{array}$ & Inedible & $\begin{array}{l}\text { NUBOT-TA- } \\
\text { LU-50 }\end{array}$ \\
\hline $\begin{array}{l}\text { Amanita cokeri E.- } \\
\text { J. Gilbert \& Kühner } \\
\text { ex E.-J. Gilbert }\end{array}$ & Amanitaceae & $\begin{array}{l}\text { Under sub-tropical semi- } \\
\text { evergreen forests, uncommon. }\end{array}$ & Poisonous & $\begin{array}{l}\text { NUBOT-TA- } \\
\text { AC-45 }\end{array}$ \\
\hline $\begin{array}{l}\text { Amanita flavoconia } \\
\text { G.F. Atk. }\end{array}$ & Amanitaceae & $\begin{array}{l}\text { Found to grow with oaks and } \\
\text { other hardwoods including } \\
\text { conifers; growing alone or } \\
\text { scattered, uncommon. }\end{array}$ & Poisonous & $\begin{array}{l}\text { NUBOT-TA- } \\
\text { AF-128 }\end{array}$ \\
\hline Amanita fulva Fr. & Amanitaceae & $\begin{array}{l}\text { Under Castanopsis and } \\
\text { Lithocarpus species in sub- } \\
\text { tropical forests, common. }\end{array}$ & Inedible & $\begin{array}{l}\text { NUBOT-TA- } \\
\text { AF-60 }\end{array}$ \\
\hline $\begin{array}{l}\text { Amanita phalloides } \\
\text { (Vaill. ex Fr.) Link }\end{array}$ & Amanitaceae & $\begin{array}{l}\text { Under sub-tropical semi- } \\
\text { evergreen forests, common. }\end{array}$ & Poisonous & $\begin{array}{l}\text { NUBOT-TA- } \\
\text { AP-39 }\end{array}$ \\
\hline $\begin{array}{l}\text { Amanita } \\
\text { rubrovolvota S. } \\
\text { Imai }\end{array}$ & Amanitaceae & $\begin{array}{l}\text { Grows in association with } \\
\text { Castanopsis, Lithocarpus and } \\
\text { Rhododendron, Quercus, etc, } \\
\text { occasional. }\end{array}$ & Inedible & $\begin{array}{l}\text { NUBOT-TA- } \\
\text { AR-66 }\end{array}$ \\
\hline $\begin{array}{l}\text { Amanita } \\
\text { strobiliformis } \\
\text { (Paulet ex Vittad.) } \\
\text { Bertill }\end{array}$ & Amanitaceae & $\begin{array}{l}\text { Under sub-tropical semi- } \\
\text { evergreen forest trees, } \\
\text { uncommon. }\end{array}$ & Edible & $\begin{array}{l}\text { NUBOT-TA- } \\
\text { AS-19 }\end{array}$ \\
\hline $\begin{array}{l}\text { Amanita vaginata } \\
\text { (Bull.) Lam. }\end{array}$ & Amanitaceae & $\begin{array}{l}\text { Grows singly or numerous and } \\
\text { found mostly in hardwoods and } \\
\text { coniferous, common. }\end{array}$ & Inedible & $\begin{array}{l}\text { NUBOT-TA- } \\
\text { AV-33 }\end{array}$ \\
\hline $\begin{array}{l}\text { Amanita virosa } \\
\text { (Fr.) Bertill. }\end{array}$ & Amanitaceae & In mixed forests, occasional. & Poisonous & $\begin{array}{l}\text { NUBOT-TA- } \\
\text { AV-81 }\end{array}$ \\
\hline $\begin{array}{l}\text { Suillus luteus (L.) } \\
\text { Roussel }\end{array}$ & Suillaceae & $\begin{array}{l}\text { Under coniferous especially pine, } \\
\text { frequent. }\end{array}$ & Edible & $\begin{array}{l}\text { NUBOT-TA- } \\
\text { SL-46 }\end{array}$ \\
\hline $\begin{array}{l}\text { Suillus pictus } \\
\text { (Peck) A.H. Sm. \& } \\
\text { Thiers }\end{array}$ & Suillaceae & $\begin{array}{l}\text { Under sub-tropical semi- } \\
\text { evergreen forests, frequent. }\end{array}$ & Edible & $\begin{array}{l}\text { NUBOT-TA- } \\
\text { SP-49 }\end{array}$ \\
\hline Suillus sp. & Suillaceae & $\begin{array}{l}\text { Under Castanopsis and } \\
\text { Lithocarpus in sub-tropical } \\
\text { forests }\end{array}$ & Inedible & $\begin{array}{l}\text { NUBOT-TA- } \\
\text { S-88 }\end{array}$ \\
\hline $\begin{array}{l}\text { Laccaria laccata } \\
\text { (Scop.) Cooke }\end{array}$ & Hydnangiaceae & $\begin{array}{l}\text { Found in troops in woods, } \\
\text { common. }\end{array}$ & Edible & $\begin{array}{l}\text { NUBOT-TA- } \\
\text { LL-109 }\end{array}$ \\
\hline $\begin{array}{l}\text { Laccaria tortilis } \\
\text { (Bolton) Cooke }\end{array}$ & Hydnangiaceae & $\begin{array}{l}\text { On bare soil in damp woods, } \\
\text { common. }\end{array}$ & Edible & $\begin{array}{l}\text { NUBOT-TA- } \\
\text { LT-51 }\end{array}$ \\
\hline $\begin{array}{l}\text { Crepidotus } \\
\text { applanatus (Pers.) } \\
\text { P. Kumm. }\end{array}$ & Inocybaceae & $\begin{array}{l}\text { On fallen branches and twigs in } \\
\text { semi-evergreen forests, } \\
\text { occasional. }\end{array}$ & Inedible & $\begin{array}{l}\text { NUBOT-TA- } \\
\text { CA-54 }\end{array}$ \\
\hline $\begin{array}{l}\text { Crepidotus luteolus } \\
\text { Sacc. }\end{array}$ & Inocybaceae & $\begin{array}{l}\text { Grows on fallen branches and } \\
\text { twigs in semi-evergreen forests, } \\
\text { occasional. }\end{array}$ & Inedible & $\begin{array}{l}\text { NUBOT-TA- } \\
\text { CL-56 }\end{array}$ \\
\hline $\begin{array}{l}\text { Crepidotus mollis } \\
\text { (Schaeff.) Staude }\end{array}$ & Inocybaceae & $\begin{array}{l}\text { In groups or overlapping tiers on } \\
\text { fallen branches and tree trunks, } \\
\text { common. }\end{array}$ & Inedible & $\begin{array}{l}\text { NUBOT-TA- } \\
\text { CM-29 }\end{array}$ \\
\hline
\end{tabular}


Table 1 Continued.

\begin{tabular}{|c|c|c|c|c|}
\hline Mushroom Species & Family & Habitat and Availability Status & Edibility & $\begin{array}{l}\text { Accession } \\
\text { No. } \\
\end{array}$ \\
\hline $\begin{array}{l}\text { Crepidotus } \\
\text { variabilis (Pers.) P. } \\
\text { Kumm. }\end{array}$ & Inocybaceae & $\begin{array}{l}\text { Found in groups on fallen tree } \\
\text { trunks, twigs and dead stumps, } \\
\text { frequent. }\end{array}$ & Inedible & $\begin{array}{l}\text { NUBOT-TA- } \\
\text { CV-133 }\end{array}$ \\
\hline $\begin{array}{l}\text { Pholiota aurivella } \\
\text { (Batsch) P. Kumm. }\end{array}$ & Strophariaceae & $\begin{array}{l}\text { Found in clusters on living and } \\
\text { dead tree stumps of conifers and } \\
\text { hardwoods, occasional. }\end{array}$ & Inedible & $\begin{array}{l}\text { NUBOT-TA- } \\
\text { PA-113 }\end{array}$ \\
\hline $\begin{array}{l}\text { Psilocybe } \\
\text { semilanceata (Fr.) } \\
\text { P. Kumm. }\end{array}$ & Strophariaceae & $\begin{array}{l}\text { Found in dense clusters in woods, } \\
\text { common, hallucinogenic, } \\
\text { common. }\end{array}$ & Inedible & $\begin{array}{l}\text { NUBOT-TA- } \\
\text { PS-120 }\end{array}$ \\
\hline $\begin{array}{l}\text { Hypholoma } \\
\text { capnoides (Fr.) P. } \\
\text { Kumm. }\end{array}$ & Strophariaceae & $\begin{array}{l}\text { Under sub-tropical semi- } \\
\text { evergreen forests, common. }\end{array}$ & Inedible & $\begin{array}{l}\text { NUBOT-TA- } \\
\text { HC-58 }\end{array}$ \\
\hline $\begin{array}{l}\text { Pholiota } \\
\text { highlandensis } \\
\text { (Peck) Quadr. \& } \\
\text { Lunghini }\end{array}$ & Strophariaceae & $\begin{array}{l}\text { Found on ground among debris in } \\
\text { forests, occasional. }\end{array}$ & Inedible & $\begin{array}{l}\text { NUBOT-TA- } \\
\text { PH-126 }\end{array}$ \\
\hline $\begin{array}{l}\text { Aleuria aurantia } \\
\text { (Pers.) Fuckel }\end{array}$ & $\begin{array}{l}\text { Pyronematacea } \\
\text { e }\end{array}$ & $\begin{array}{l}\text { Grows in groups on soil amongst } \\
\text { grasses or on bare soil or at } \\
\text { roadside, common. }\end{array}$ & Edible & $\begin{array}{l}\text { NUBOT-TA- } \\
\text { AA-62 }\end{array}$ \\
\hline $\begin{array}{l}\text { Octospora rutilans } \\
\text { (Fr.) Dennis \& } \\
\text { Itzerott }\end{array}$ & $\begin{array}{l}\text { Pyronematacea } \\
\text { e }\end{array}$ & $\begin{array}{l}\text { Found in clusters amongst } \\
\text { mosses, uncommon. }\end{array}$ & Inedible & $\begin{array}{l}\text { NUBOT-TA- } \\
\text { OR-117 }\end{array}$ \\
\hline $\begin{array}{l}\text { Otidea alutacea } \\
\text { (Pers.) Massee }\end{array}$ & $\begin{array}{l}\text { Pyronematacea } \\
\text { e }\end{array}$ & $\begin{array}{l}\text { Found in clusters on ground in } \\
\text { woods, uncommon. }\end{array}$ & Inedible & $\begin{array}{l}\text { NUBOT-TA- } \\
\text { OA-91 }\end{array}$ \\
\hline $\begin{array}{l}\text { Clavulina } \\
\text { coralloides (L.) J. } \\
\text { Schröt. }\end{array}$ & Clavulinaceae & $\begin{array}{l}\text { Found with deciduous or } \\
\text { coniferous trees, uncommon. }\end{array}$ & Inedible & $\begin{array}{l}\text { NUBOT-TA- } \\
\text { CC-94 }\end{array}$ \\
\hline $\begin{array}{l}\text { Pleurotus } \\
\text { citrinopileatus } \\
\text { Singer }\end{array}$ & Pleurotaceae & $\begin{array}{l}\text { In clusters on cut timber and } \\
\text { fallen logs, frequent. }\end{array}$ & Edible & $\begin{array}{l}\text { NUBOT-TA- } \\
\text { PC-75 }\end{array}$ \\
\hline $\begin{array}{l}\text { Pleurotus ostreatus } \\
\text { (Jacq) P. Kumm }\end{array}$ & Pleurotaceae & $\begin{array}{l}\text { Grows in clusters on dead or } \\
\text { leaving trees, common. }\end{array}$ & Edible & $\begin{array}{l}\text { NUBOT-TA- } \\
\text { PO-65 }\end{array}$ \\
\hline $\begin{array}{l}\text { Pleurotus } \\
\text { pulmonarius (Fr.) } \\
\text { Quél. }\end{array}$ & Pleurotaceae & $\begin{array}{l}\text { In clusters on cut timber and } \\
\text { fallen logs, frequent. }\end{array}$ & Edible & $\begin{array}{l}\text { NUBOT-TA- } \\
\text { PP-28 }\end{array}$ \\
\hline $\begin{array}{l}\text { Termitomyces } \\
\text { eurhizus (Berk.) R. } \\
\text { Heim }\end{array}$ & Lyophyllaceae & $\begin{array}{l}\text { Grows in groups on ground near } \\
\text { termite mounts, common. }\end{array}$ & Edible & $\begin{array}{l}\text { NUBOT-TA- } \\
\text { TE-71 }\end{array}$ \\
\hline $\begin{array}{l}\text { Termitomyces } \\
\text { heimii Natarajan }\end{array}$ & Lyophyllaceae & $\begin{array}{l}\text { Grows on termite mounds and } \\
\text { clayey soil, common. }\end{array}$ & Edible & $\begin{array}{l}\text { NUBOT-TA- } \\
\text { TH-64 }\end{array}$ \\
\hline $\begin{array}{l}\text { Termitomyces } \\
\text { microcarpus (Berk. } \\
\text { \& Broome) R. } \\
\text { Heim }\end{array}$ & Lyophyllaceae & $\begin{array}{l}\text { Grows in groups on termite } \\
\text { mounts, frequent. }\end{array}$ & Edible & $\begin{array}{l}\text { NUBOT-TA- } \\
\text { TM-76 }\end{array}$ \\
\hline Termitomyces sp. & Lyophyllaceae & $\begin{array}{l}\text { Grows in groups on termite } \\
\text { mounts, frequent. }\end{array}$ & Edible & $\begin{array}{l}\text { NUBOT-TA- } \\
\text { T-77 }\end{array}$ \\
\hline $\begin{array}{l}\text { Phallus impudicus } \\
\text { L. }\end{array}$ & Phallaceae & $\begin{array}{l}\text { Found singly or scattered } \\
\text { associated with rotting wood in } \\
\text { gardens and woods, common. }\end{array}$ & Inedible & $\begin{array}{l}\text { NUBOT-TA- } \\
\text { PI-125 }\end{array}$ \\
\hline $\begin{array}{l}\text { Phallus indusiatus } \\
\text { Vent. }\end{array}$ & Phallaceae & $\begin{array}{l}\text { Grows singly on soil in woods, } \\
\text { common. }\end{array}$ & Unknown & $\begin{array}{l}\text { NUBOT-TA- } \\
\text { PI-84 }\end{array}$ \\
\hline $\begin{array}{l}\text { Bulgaria inquinans } \\
\text { (Pers.) Fr. }\end{array}$ & Bulgariaceae & $\begin{array}{l}\text { Found in clusters on fallen trunks } \\
\text { like Lithocarpus, uncommon. }\end{array}$ & Inedible & $\begin{array}{l}\text { NUBOT-TA- } \\
\text { BI-121 }\end{array}$ \\
\hline
\end{tabular}


Table 1 Continued.

\begin{tabular}{|c|c|c|c|c|}
\hline Mushroom Species & Family & Habitat and Availability Status & Edibility & $\begin{array}{l}\text { Accession } \\
\text { No. }\end{array}$ \\
\hline $\begin{array}{l}\text { Hypholoma } \\
\text { fasciculare (Huds.) } \\
\text { P.Kumm. }\end{array}$ & $\begin{array}{l}\text { Hymenogastrac } \\
\text { eae }\end{array}$ & $\begin{array}{l}\text { Found in dense clusters on trunks } \\
\text { or stumps with deciduous or } \\
\text { coniferous trees, common. }\end{array}$ & Inedible & $\begin{array}{l}\text { NUBOT-TA- } \\
\text { HF-96 }\end{array}$ \\
\hline $\begin{array}{l}\text { Helvella crispa } \\
\text { (Scop.) Fr. }\end{array}$ & Helvellaceae & $\begin{array}{l}\text { Found in deciduous woods, } \\
\text { uncommon. }\end{array}$ & Poisonous & $\begin{array}{l}\text { NUBOT-TA- } \\
\text { HC-90 }\end{array}$ \\
\hline $\begin{array}{l}\text { Sparassis crispa } \\
\text { (Wulfen) Fr. }\end{array}$ & Sparassidaceae & $\begin{array}{l}\text { Found at the base of tree trunks, } \\
\text { uncommon. }\end{array}$ & Edible & $\begin{array}{l}\text { NUBOT-TA- } \\
\text { SC-92 }\end{array}$ \\
\hline $\begin{array}{l}\text { Armillaria mellea } \\
\text { (Vahl) P. Kumm. }\end{array}$ & $\begin{array}{l}\text { Physalacriacea } \\
\text { e }\end{array}$ & $\begin{array}{l}\text { Found in dense clusters on or } \\
\text { around tree trunks or stumps with } \\
\text { deciduous or coniferous trees, } \\
\text { common. }\end{array}$ & Edible & $\begin{array}{l}\text { NUBOT-TA- } \\
\text { AM-127 }\end{array}$ \\
\hline $\begin{array}{l}\text { Flammulina } \\
\text { velutipes (Curtis) } \\
\text { Singer }\end{array}$ & $\begin{array}{l}\text { Physalacriacea } \\
\text { e }\end{array}$ & $\begin{array}{l}\text { Found to grow in clusters on } \\
\text { decaying trees, survive cold } \\
\text { weather, common }\end{array}$ & Edible & $\begin{array}{l}\text { NUBOT-TA- } \\
\text { FV-97 }\end{array}$ \\
\hline $\begin{array}{l}\text { Xerula radicata } \\
\text { (Relhan) Dörfelt }\end{array}$ & $\begin{array}{l}\text { Physalacriacea } \\
\text { e }\end{array}$ & $\begin{array}{l}\text { Found under grasses or leaf } \\
\text { debris, common. }\end{array}$ & Inedible & $\begin{array}{l}\text { NUBOT-TA- } \\
\text { XR-110 }\end{array}$ \\
\hline $\begin{array}{l}\text { Hemimycena lactea } \\
\text { (Pers.) Singer }\end{array}$ & Mycenaceae & $\begin{array}{l}\text { Found in large groups in forest, } \\
\text { occasional. }\end{array}$ & Inedible & $\begin{array}{l}\text { NUBOT-TA- } \\
\text { HL-99 }\end{array}$ \\
\hline $\begin{array}{l}\text { Mycena erubescens } \\
\text { Höhn. }\end{array}$ & Mycenaceae & $\begin{array}{l}\text { Under semi-evergreen forests } \\
\text { including pine, occasional. }\end{array}$ & Inedible & $\begin{array}{l}\text { NUBOT-TA- } \\
\text { ME-55 }\end{array}$ \\
\hline $\begin{array}{l}\text { Mycena galopus } \\
\text { (Pers.) P. Kumm. }\end{array}$ & Mycenaceae & $\begin{array}{l}\text { Found under leaf litter, dead and } \\
\text { old tree stumps in woods, } \\
\text { common. }\end{array}$ & Inedible & $\begin{array}{l}\text { NUBOT-TA- } \\
\text { MG-111 }\end{array}$ \\
\hline $\begin{array}{l}\text { Mycena inclinata } \\
\text { (Fr.) Quél. }\end{array}$ & Mycenaceae & $\begin{array}{l}\text { Found in dense tufts or clusters } \\
\text { on tree stumps, common. }\end{array}$ & Inedible & $\begin{array}{l}\text { NUBOT-TA- } \\
\text { MI-98 }\end{array}$ \\
\hline $\begin{array}{l}\text { Xeromphalina } \\
\text { kauffmanii A.H. } \\
\text { Sm. }\end{array}$ & Mycenaceae & $\begin{array}{l}\text { Found in clusters on coniferous } \\
\text { stumps and logs, frequent. }\end{array}$ & Inedible & $\begin{array}{l}\text { NUBOT-TA- } \\
\text { XK-139 }\end{array}$ \\
\hline $\begin{array}{l}\text { Entoloma murrayi } \\
\text { (Berk. \& } \\
\text { M.A.Curtis) Sacc. }\end{array}$ & $\begin{array}{l}\text { Entolomatacea } \\
\mathrm{e}\end{array}$ & $\begin{array}{l}\text { Found singly or in small groups } \\
\text { on ground in litter or humus with } \\
\text { conifers and hardwoods, } \\
\text { uncommon. }\end{array}$ & Inedible & $\begin{array}{l}\text { NUBOT-TA- } \\
\text { EM-119 }\end{array}$ \\
\hline $\begin{array}{l}\text { Pseudohydnum } \\
\text { gelatinosum (Scop.) } \\
\text { P. Karst. }\end{array}$ & Exidiaceae & $\begin{array}{l}\text { Found under rotting and decaying } \\
\text { tree stumps, common. }\end{array}$ & Edible & $\begin{array}{l}\text { NUBOT-TA- } \\
\text { PG-124 }\end{array}$ \\
\hline $\begin{array}{l}\text { Cookeina } \\
\text { tricholoma (Mont.) } \\
\text { Kuntze }\end{array}$ & $\begin{array}{l}\text { Sarcoscyphace } \\
\text { ae }\end{array}$ & $\begin{array}{l}\text { Found in clusters on trunks or } \\
\text { stumps with deciduous or } \\
\text { coniferous trees, uncommon. }\end{array}$ & Inedible & $\begin{array}{l}\text { NUBOT-TA- } \\
\text { CT-93 }\end{array}$ \\
\hline Sarcoscypha sp. & $\begin{array}{l}\text { Sarcoscyphace } \\
\text { ae }\end{array}$ & $\begin{array}{l}\text { Found on dead tree trunks and old } \\
\text { stems, uncommon }\end{array}$ & Inedible & $\begin{array}{l}\text { NUBOT-TA- } \\
\text { S-135 }\end{array}$ \\
\hline $\begin{array}{l}\text { Ductifera sucina } \\
\text { (Möller) K.Wells }\end{array}$ & Incertae sedis & $\begin{array}{l}\text { Found on decayed wood of } \\
\text { hardwoods and old stems, } \\
\text { uncommon. }\end{array}$ & Inedible & $\begin{array}{l}\text { NUBOT-TA- } \\
\text { DS-141 }\end{array}$ \\
\hline
\end{tabular}



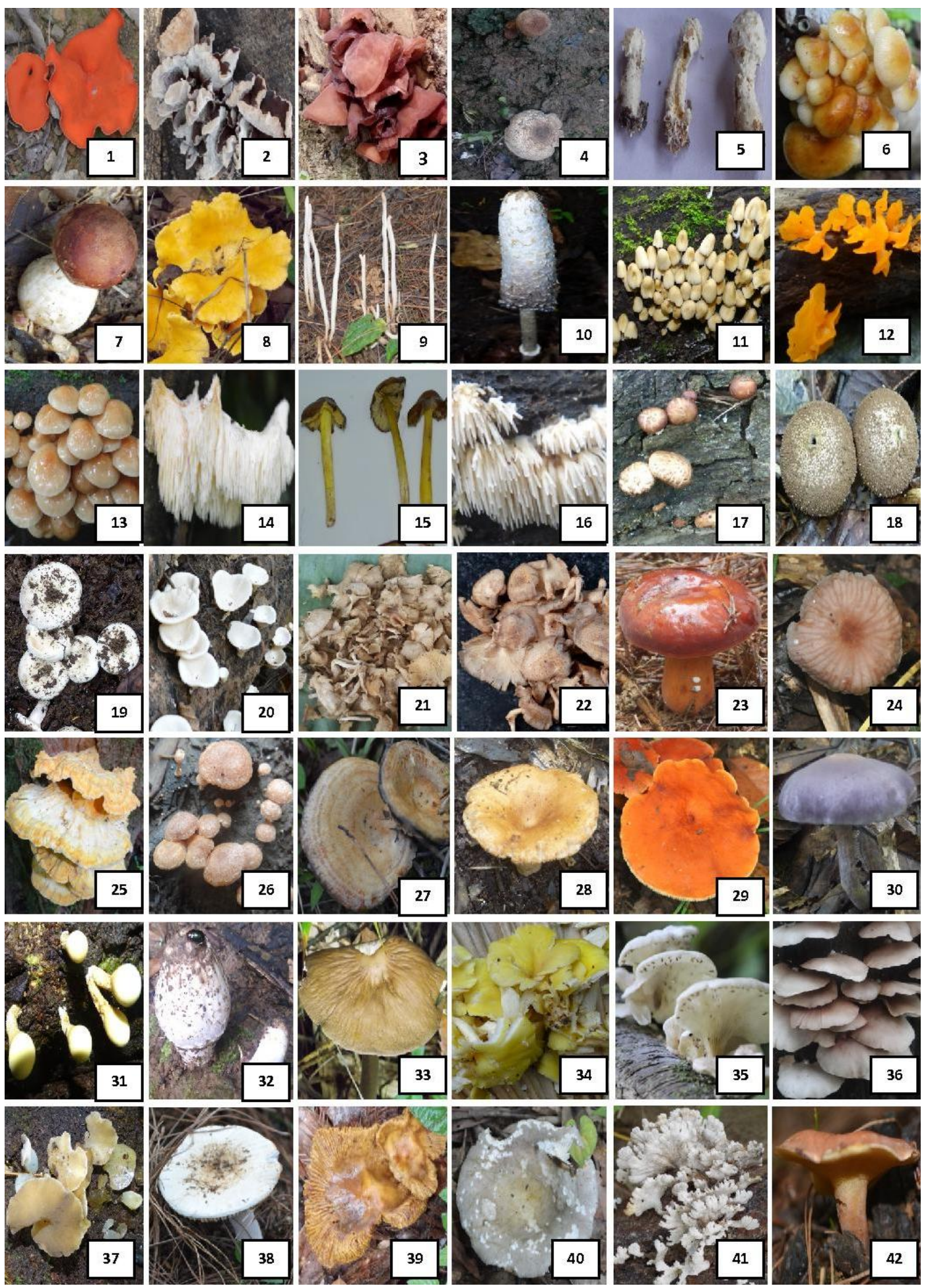

Fig. 2 - Wild edible mushrooms of Nagaland: 1 Aleuria aurantia. 2 Auricularia polytricha. 3 Auricularia auricula judae. 4 Agaricus augustus. 5 Amanita strobiliformis. 6 Armillaria mellea. 7 Boletus edulis. 8 Cantharellus cibarius. 9 Clavaria fragilis. 10 Coprinus comatus. 11 Coprinellus micaceus. 12 Dacryopinax spathularia. 13 Flammulina velutipes. 14 Hericium cirrhatum. 15 Hygrocybe conica. 16 Hericium erinaceus. 17 Lentinula edodes. 18 Lycoperdon perlatum. 19 Lactifluus piperatus. 20 Lentinus sajor caju. 21 Lentinus squarrosulus. 22 Lentinus tigrinus. 23 Lactifluus volemus. 24 Laccaria tortilis. 25 Laetiporus sulphureus. 26 Laccaria laccata. 27 Lactarius deliciosus. 28 Lactarius deterrimus. 29 Lactarius subdulcis. 30 Lepista nuda. 31 Lycoperdon pyriforme. 32 Macrolepiota albuminosa. 33 Melanoleuca grammopodia. 34 Pleurotus citrinopileatus. 35 Pleurotus ostreatus. 36 Pleurotus pulmonarius. 37 Pseudohydnum 
gelatinosum. 38 Russula ochroleuca. 39 Russula puellaris. 40 Russula heterophylla, 41 Schizophyllum commune. 42 Suillus luteus.
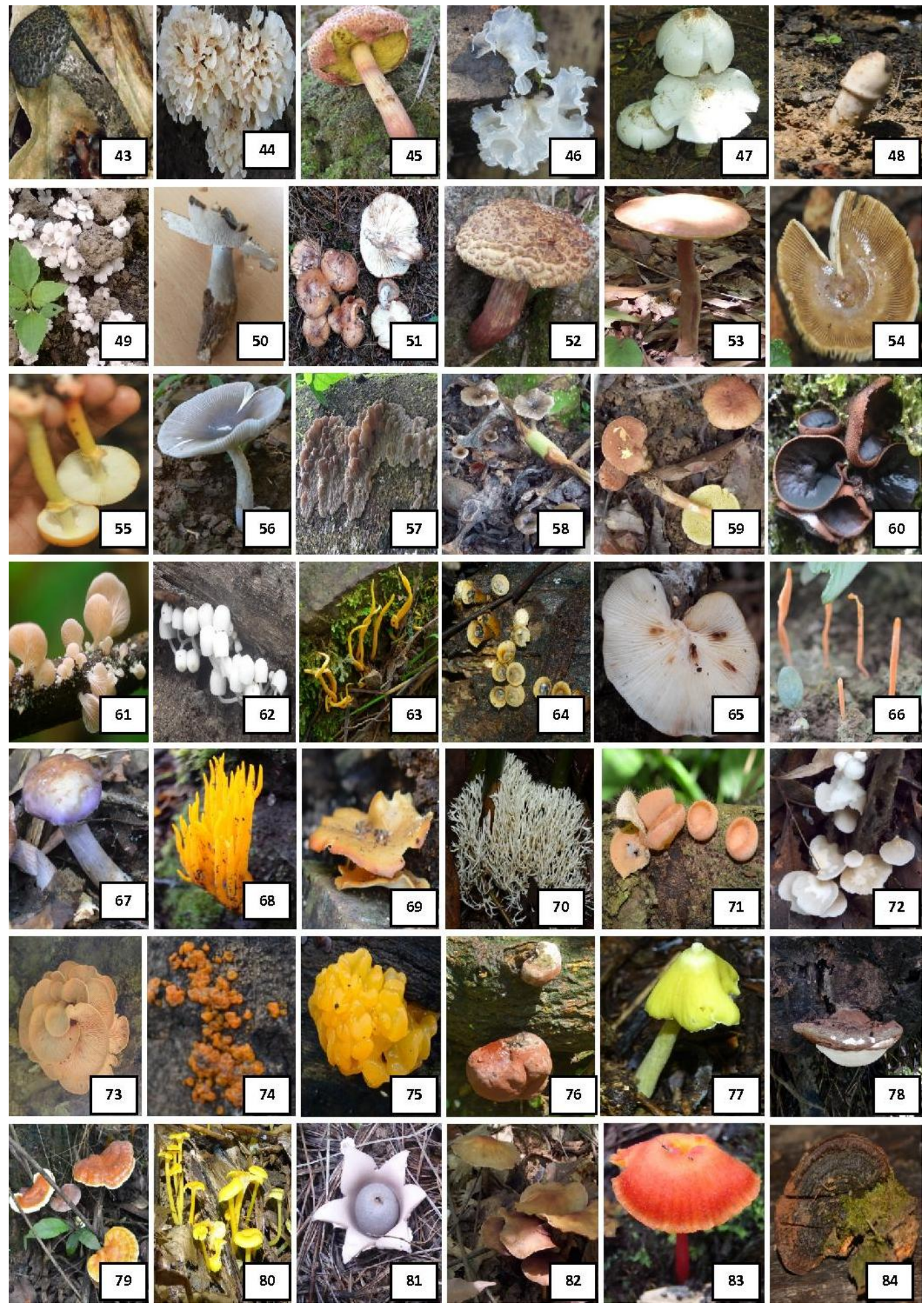

Fig. 3 - Wild mushrooms of Nagaland (Edible - 43-52; Inedible - 53-84): 43 Strobilomyces strobilaceus. 44 Sparassis crispa. 45 Suillus pictus. 46 Tremella fuciformis. 47 Termitomyces heimii. 48 Termitomyces eurrhizus. 49 Termitomyces microcarpus. 50 Termitomyces sp. 51 Tricholoma imbricatum. 52 Xerocomellus chrysenteron. 53 Aureoboletus auriporus. 54 Amanita fulva. 55 Amanita rubrovolvata. 56 Amanita vaginata. 57 Auricularia mesenterica. 58 Arrhenia onisca. 59 Boletus sp. 60 Bulgaria inquinans. 61 Crepidotus applanatus. 62 Coprinus disseminatus. 63 Clavulinopsis fusiformis. 64 Crucibulum leave. 65 Crepidotus luteolus. 66 Cordyceps militaris. 67 Cortinarius purpurascens. 68 Calocera viscosa. 69 Cerioporus 
leptocephalus. 70 Clavulina coralloides. 71 Cookeina tricholoma. 72 Crepidotus mollis. 73 Crepidotus variabilis. 74 Dacrymyces stillatus. 75 Dacrymyces chrysospermus. 76 Daldinia concentrica. 77 Entoloma murrayi. 78 Ganoderma applanatum. 79 Ganoderma lucidum. 80 Gloioxanthomyces nitidus. 81 Geastrum sp. 82 Hypholoma capnoides. 83 Hygrocybe miniata. 84 Hymenochaete rubiginosa.

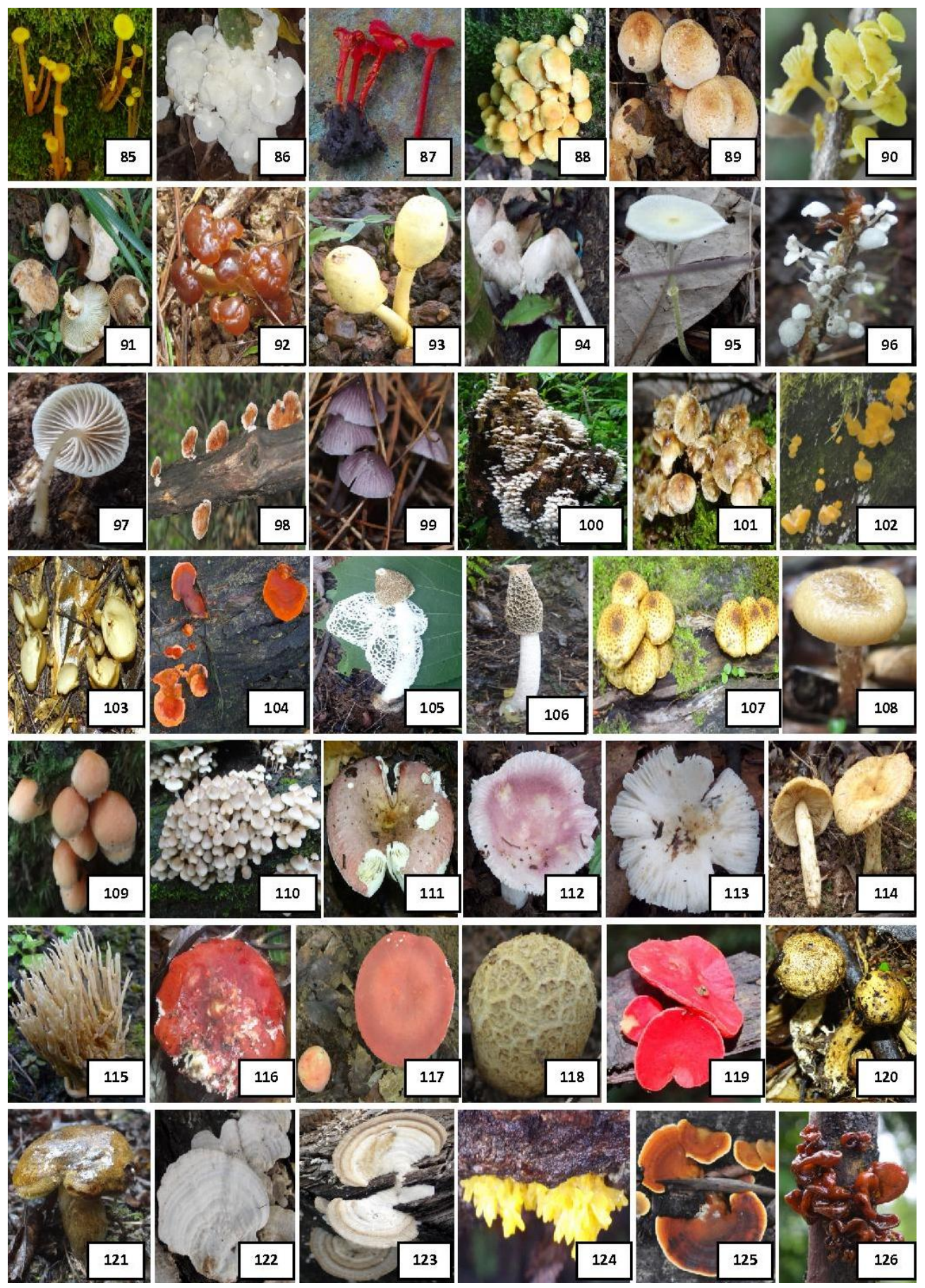

Fig. 4 - Wild mushrooms of Nagaland (Inedible): 85 Hygrocybe vitellina. 86 Hemimycena lactea. 87 Hygrocybe cantharellus. 88 Hypholoma fasciculare. 89 Lepiota cristata. 90 Lichenomphalia umbellifera. 91 Lactifluus glaucescens. 92 Leotia lubrica. 93 Leucocoprinus birnbaumii. 94 Leucocoprinus brebissonii. 95 Leucocoprinus fragilissimus. 96 Marasmiellus candidus. 97 Marasmiellus ramealis. 98 Microporus xanthopus. 99 Mycena erubescens. 100 Mycena galopus. 101 Mycena inclinata. 102 Octospora rutilans. 103 Otidea alutacea. 104 Pcynoporus cinnabarinus. 105 Phallus indusiatus. 106 Phallus impudicus. 107 Pholiota aurivella. 108 Pholiota 
highlandensis. 109 Psathyrella piluliformis. 110 Psilocybe semilanceata. 111 Russula cyanoxantha. 112 Russula fragilis. 113 Russula natarajanii. 114 Russula senecis. 115 Ramaria stricta. 116 Russula rosea. 117 Russula sp. 118 Scleroderma citrinum. 119 Sarcoscypha sp. 120 Scleroderma areolatum. 121 Suillus sp. 122 Trametes gibbosa. 123 Trametes hirsuta. 124 Tremella mesenterica. 125 Trametes versicolor. 126 Tremella foliacea.
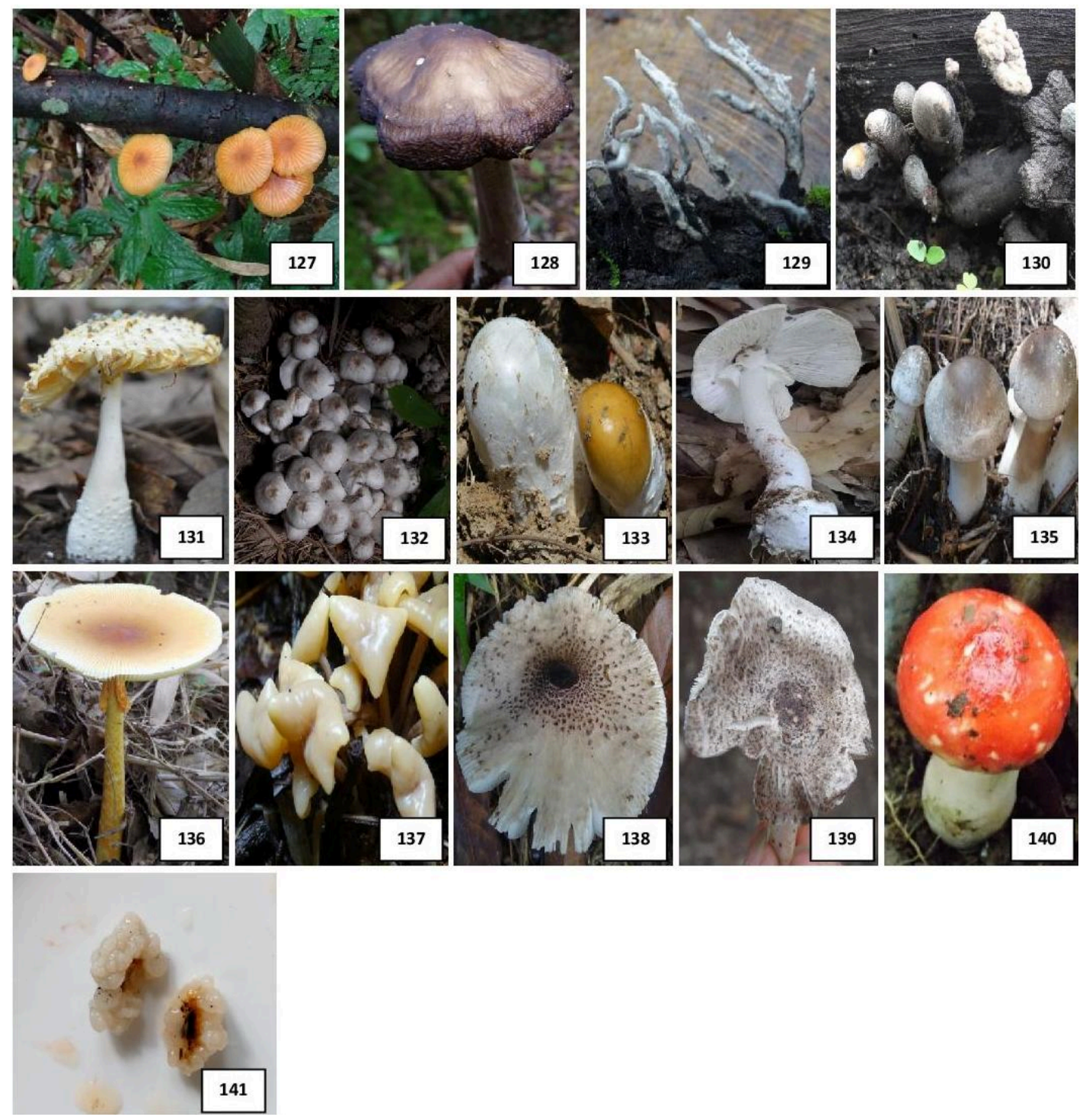

Fig. 5 - Wild mushrooms of Nagaland (Inedible - 127-130; Poisonous - 131-140): 127 Xeromphalina kauffmanii. 128 Xerula radicata. 129 Xylaria hypoxylon. 130 Xylaria polymorpha. 131 Amanita cokeri. 132 Agaricus moelleri. 133 Amanita phalloides. 134 Amanita virosa. 135 Agaricus xanthodermus. 136 Amanita flavoconia. 137 Helvella crispa. 138 Lepiota felina. 139 Lepiota lilacea. 140 Russula nobilis. 141 Ductifera sucina.

\section{Conclusion}

The present investigation shows the wild mushroom diversity of Nagaland, India. The database of wild mushrooms created in this study will help future researchers to make further indepth study of the potential mushrooms available. Mushrooms are a boon to mankind and should be judiciously exploited for societal benefits across the globe.

\section{Acknowledgement}

The authors are thankful to Department of Biotechnology, Ministry of Science and Technology, Government of India, New Delhi for facilities provided through Institutional Biotech 
Hub and University Grants Commission, New Delhi sponsored SAP (DRS-III) programme. Toshinungla Ao is thankful to University Grants Commission, New Delhi, India for UGC-BSR fellowship for her $\mathrm{Ph}$. D. programme. Facilities used from DST-FIST programme are duly acknowledged.

\section{Conflicts of interests}

Authors declare that there are no conflicts of interests of any kind.

\section{References}

Andrew EE, Kinge TR, Tabi EM, Thiobal N, Mih AM. 2013 - Diversity and distribution of macrofungi (mushrooms) in the Mount Cameroon Region. Journal of Environmental Microbiology 3, 318-334.

Ao T, Deb CR. 2019 - Nutritional and antioxidant potential of some wild edible mushrooms of Nagaland, India. Journal of Food Science and Technology 56(2), 1084-1089. https://doi.org/10.1007/s13197-018-03557-w.

Ao T, Deb CR, Khruomo N. 2016 - Wild edible mushrooms of Nagaland, India: a potential food resource. Journal of Experimental Biology and Agricultural Science 4(1), 59-65.

Atri NS, Saini SS, Saini MK. 1997 - Studies on genus Russula Pers. from North Western Himalayas. Mushroom Research 6(1), 1-6.

Bhattacharjee J, Bhattacharjee D, Paul T, Kumar A, Chowdhury S. 2015 - Diversity of mushrooms in Indo-Bangladesh region of North-East India. Journal of the Andaman Science Association. 19(1), 75-82.

Boa E. 2004 - Wild edible fungi: a global overview of their use and importance to people. Nonwood forest products. Food and Agricultural Organization Publications, Rome.

Bonatti M, Karnopp P, Soares HM, Furlan SA. 2004 - Evaluation of Pleurotus ostreatus and Pleurotus sajor-caju nutritional characteristics when cultivated in different lignocellulosic wastes. Food Chemistry 88(3), 425-428.

Chang ST, Miles PG. 1992 - Mushroom biology - A new discipline. Mycologist 6, 64-65.

Chang ST, Miles PG. 2004 - Mushrooms cultivation, nutritional value, medicinal effect and environmental impact. Mycologist 6, 64-65.

Christensen CM. 1943 - Common Edible Mushrooms. The University of Minnesota Press. Minneapolis.

Crawshay R .1930 - The spore ornamentation of the Russulales. Baillière, Tindall \& Cox. Cornell University.

Das K. 2009 - Mushrooms of Sikkim I: Barsey Rhododendron Sanctuary. Sikkim State Biodiversity Board and Botanical Survey of India.

Das K, Sharma JR. 2005 - Russulaceae of Kumaon Himalaya. BSI and Ministry of Environment and Forests, Govt. of India.

Diez JM, James TY, Mcmunn M, Ibanez I. 2013 - Predicting species-specific responses of fungi to climatic variation using historical records. Global Change Biology 19, 3145-3154.

Ferreira ICFR, Baptista P, Boas VM, Barros L. 2007 - Free-radical scavenging capacity and reducing power of wild edible mushrooms from northeast Portugal. Food Chemistry 100, 1511-1516.

Lakhanpal TN, Rana M. 2005 - Medicinal and nutraceutical genetic resources of mushrooms. Plant Genetic Resources: Characterization and Utilization 3, 288-303.

Manoharachary C, Sridhar K, Singh R, Adholeya A et al. 2005 - Fungal biodiversity: distribution, conservation and prospecting of fungi from India. Current Science 89(1), 58-71.

Manzi P, Gambelli L, Marconi S, Vivanti V, Pizzoferrato L - 1999. Nutrients in edible mushrooms: an inter-species comparative study. Food Chemistry 65, 477-482.

Pegler DN. 1983 - The genus Lentinus ‘A World Monograph’. Kew Bulletin Additional Series X. Royal Botanic Garden Kew. 
Philips R. 2006 - Mushrooms. Pan Macmillan, London.

Mortimer PE, Xu J, Karunarathna SC, Hyde KD. 2014 - Mushrooms for Trees and People. A Field Guide to Useful Mushrooms of the Mekong Region. World Agroforestry Centre, East and Central Asia, Kunming, 650201 PR China.

Roy A, De AB. 1996 - Polyporaceae of India. International Book Distributors, Dehradun, India.

Sysouphanthong P, Thongkantha S, Zhao RL, Soytong K, Hyde KD. 2010 - Mushroom diversity in sustainable shade tea forest and the effect of fire damage. Biodiversity Conservation 19, 1401-1415.

Tibuhwa DD. 2013 - Wild mushroom - an underutilized healthy food resource and income generator: experience from Tanzania rural areas. Journal of Ethnobiology and Ethnomedicine 9(49), 1-13.

Wani BA, Bodha RH, Wani AH. 2010 - Nutritional and medicinal importance of mushrooms. Journal of Medicinal Plant Research 4(24), 2598-2604.

Wasser SP, Weis A. 1999 - Medicinal properties of substances occurring in higher basidiomycetes mushrooms: current perspective (review). International Journal of Medicinal Mushrooms 1, 31-62.

Wasser S. 2002 - Medicinal mushrooms as a source of anti-tumor and immuno-modulating polysaccharides. Applied Microbiology and Biotechnology 60(3), 258-274.

Wongchalee P, Pukahute C. 2012 - Diversity of mushrooms in dry Dipterocarp forest at Phuphan National Park, Sakon Nakhon Province. Natural Science 4(12), 1153-1160. 